\title{
Relationship between oral habits and spirometry maneuvers, in children
}

\author{
Rafaela Coelho Minsky (1) \\ Tayná Castilho( ${ }^{(1)}$ \\ Roseane Rebelo Silva Meira(2) \\ Tatiana Godoy Bobbio(3) \\ Camila Isabel Santos Schivinski(1)
}

1) Universidade do Estado de Santa Catarina, Florianópolis, Santa Catarina, Brasil.

(2) Pontifícia Universidade Católica de Campinas, Campinas, São Paulo, Brasil.

(3) Universidade Estadual de Campinas, Campinas, São Paulo, Brasil.

Conflict of interest: Nonexistent

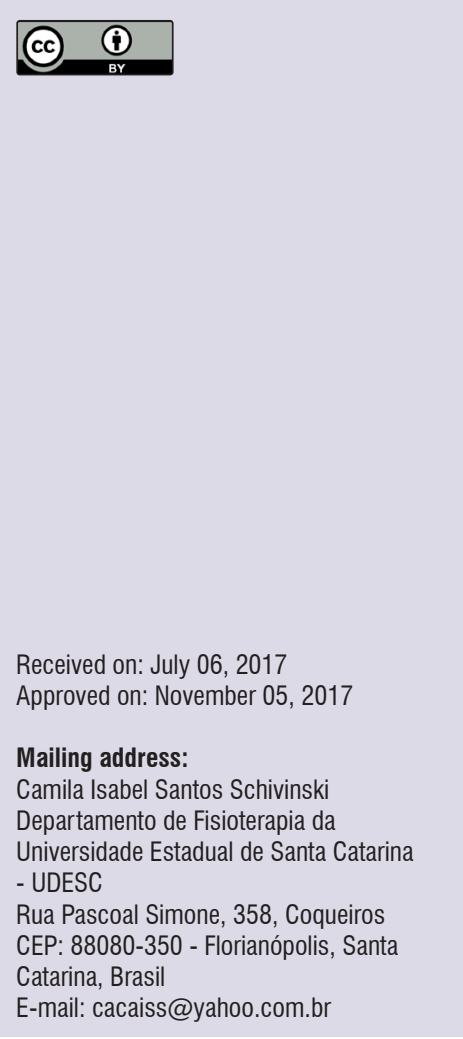

\section{ABSTRACT}

Purpose: to analyze whether deleterious oral habits can influence the number of attempts of forced spirometry maneuvers performed by healthy children.

Methods: this observational and cross-sectional analytical study included 149 healthy children aged 6-12 years attending public and private schools in Florianópolis, SC, Brazil. A validated protocol was applied for the analysis of deleterious oral habits. The children were grouped according to the number of spirometry maneuvers needed to achieve successful spirometry results, as follows: G1) children who needed 3 maneuvers; G2) 4 maneuvers; G3) 5-8 maneuvers. Data were analyzed with the KolmogorovSmirnov test and the Kruskal-Wallis test was applied to compare quantitative variables between the groups. The Chi-square test was used to assess the association between the groups and qualitative variables.

Results: there was no association between the number of attempts and the qualitative variables evaluated by the protocol. There was also no difference between the groups regarding quantitative variables for breastfeeding time, breastfeeding occurrence, use of pacifiers, and thumb sucking.

Conclusion: the presence of $\mathrm{DOH}$ did not influence the number of forced spirometry maneuvers, performed by the healthy children in this study.

Keywords: Child; Spirometry; Stomatognathic System 


\section{INTRODUCTION}

Orofacial function is the result of the integration between the central nervous system and the neuromuscular system, which includes breathing, chewing, sucking and swallowing ${ }^{1,2}$. These actions are performed by the stomatognathic system (SS), which is composed of craniofacial structures such as bones of the head, mandible, hyoid, sternum, chewing and swallowing muscles $^{3}$.

Some oral habits performed by children are considered deleterious to the SS. These include non-nutritive thumb sucking, mouth breathing, pacifiers, lower lip interposition/suction, tongue thrusting, nail biting, and jaw propulsion ${ }^{4,5}$. The persistence of deleterious oral habits $(\mathrm{DOH})$ plays a key role in causing malocclusions, orthodontic abnormalities and phonetic disorders, since they affect the growth and development of the muscles and bones of the mandible ${ }^{5-7}$. Thus, DOH may even interfere in facial aesthetics.

Spirometry is a pulmonary function test indicated for the diagnosis and monitoring of respiratory diseases, widely used in scientific research and public health ${ }^{8,9}$. When performing spirometry, the individual must place the mouthpiece on his or her tongue and seal the lips so that air does not escape during the maneuvers, which require forced expiration, muscle strength and motor coordination. For this reason, children with a history of $\mathrm{DOH}$ and possible impairment of oral functions may show difficulties in performing the test, requiring a higher number of maneuvers and possible changes in their results.

Therefore, this study aims to analyze the relationship between the presence of $\mathrm{DOH}$ and the number of attempts of forced spirometry maneuvers performed by healthy children.

\section{METHODS}

This study has been approved by the Ethics Committee of the State University of Santa Catarina under Resolution Number 1.006.003 - (CAAE: 38770314.1.0000.0118), registered in the National Research Ethics Committee involving Human Beings (CONEP). All participants provided a minor's assent document and a parent or guardian authorized their participation by signing an Informed Consent Document.

This is an observational and analytical crosssectional study, including healthy children between 6 and 12 years of age from public and private schools in the metropolitan area of Florianópolis - Santa Catarina/Brazil. Data was collected in the school site and the following individuals were selected: healthy non-athletes (children not enrolled in high-performance sports federations), non-obese children (body mass index above $25 \mathrm{~kg} / \mathrm{m}^{2}$ ) and non-undernourished (mass index lower than $\left.18.5 \mathrm{~kg} / \mathrm{m}^{2}\right)^{10}$, children with no diagnosis or history of cardiorespiratory, musculoskeletal, rheumatic, neurological diseases, auditory or visual deficits, and children with normal spirometry parameters.

Schoolchildren's health was checked according to the International Study of Asthma and Allergies in Childhood (ISAAC) ${ }^{11}$ and the analysis of health history, previously completed by a parent or tutor. Spirometry showing forced expiratory volume in one second $\left(\mathrm{FEV}_{1}\right)$ and forced vital capacity (FVC) above $80 \%$ of predicted, according to Polgar et al. (1971) 12 was also considered. A JAEGER ${ }^{\text {TM }}$ MasterScope IOS $^{\text {TM }}$ spirometer was used to record the parameters of FVC, FEV $\mathrm{F}_{1}$ and peak expiratory flow (PEF). The examination was conducted according to the American Thoracic Society's guidelines ${ }^{13}$, and forced spirometry maneuvers were performed until the values and curves were acceptable and reproducible, on a maximum of eight attempts. Children who failed to perform a valid test in this number of maneuvers were excluded.

An assessment tool based on the Orofacial Myofunctional Evaluation protocol (MBGR) ${ }^{14}$ was used to identify the presence of $\mathrm{DOH}$. This tool considers elements including time of breastfeeding and the use of the bottle; difficulties in introducing solid foods; characteristics of chewing; frequency of episodes such as choking, gastroesophageal reflux (GERD) and coughing during meal. It also controls oral habits such as use of pacifier or non-nutritive thumb sucking and speech acquisition time. These items were answered by a parent or guardian. Ultimately, it includes the participants' physical observation (made by a trained examiner) relating to lip positioning, saliva control, mobility and oral musculature tonus by means of a manual resistance (with emphasis on tongue, buccinators/orbicularis lips).

Anthropometric data was collected and assessed with an Ultra Slim W903 Wiso ${ }^{\circledR}$ digital scale (weight in $\mathrm{kg}$ ) and a Sanny ${ }^{\circledR}$ portable stadiometer (height in $\mathrm{cm})$. Next, the children underwent spirometry testing conducted by a single examiner blinded to the orofacial myofunctional disorders assessment instrument. The children were grouped according to the number of 
spirometry maneuvers needed to achieve successful spirometry results, as follows: G1) children who needed 3 maneuvers; G2) 4 maneuvers; G3) 5-8 maneuvers.

For the analyses, the variables were divided into qualitative and quantitative. Qualitative variables included: labial posture, saliva control, feeding difficulty (introduction of the cup, transition from liquid to pasty and from pasty to solid foods), characteristic of chewing (time with food inside the mouth), presence of GERD and coughing, history of non-nutritive thumb sucking or use of pacifier, bottle feeding, history of breastfeeding and speech quality. Quantitative variables included: breastfeeding period, whether the child was breastfed, used a pacifier and sucked their thumbs.
Initially, statistical analysis was conducted by evaluating distribution of data using the Kolmogorov-Smirnov test. Then, the Kruskal-Wallis test was used to compare the quantitative variables between the 3 groups. An association between the groups and the qualitative variables was observed by means of the Chi-square test. For all analyses, the significance level was set at $5 \%(p<0.05)$.

\section{RESULTS}

A total of 149 children participated in this study. They were allocated into 3 groups, whose age, gender and anthropometric measurements are described in Table 1.

Table 1. Distribution of gender, age and anthropometric data of the participants of each group

\begin{tabular}{cccc} 
& G1 & G2 & G3 \\
& $\mathbf{M}( \pm \mathbf{S D})$ & $\mathbf{M}( \pm \mathbf{S D})$ & $\mathbf{M}( \pm \mathbf{S D})$ \\
\hline Sample & 59 & 53 & 37 \\
(prevailing gender) & $(36$ girls $)$ & $(30$ boys $)$ & $(21$ girls $)$ \\
Age & $9.24( \pm 1.89)$ & $9.58( \pm 1.90)$ & $8.59( \pm 1.87)$ \\
Weight & $34.25( \pm 10.97)$ & $37.96( \pm 11.54)$ & $34.78( \pm 12.08)$ \\
Height & $1.41( \pm 0.12)$ & $1.44( \pm 0.13)$ & $1.40( \pm 0.12)$ \\
\hline
\end{tabular}

Note: G1: group 1; G2: group 2; G3: group 3; Age: in years; Weight: in kilograms; Height: in centimeters; M: mean; SD: standard deviation.

The results of the analyses of associations between the qualitative variables of orofacial function and the group are presented in Table 2. No statistically significant difference could be observed, indicating no association between the qualitative variables and the number of spirometry maneuvers.
Table 3 illustrates the results of the Kruskal-Wallis test applied for the comparison between the allocation in the 3 groups and the quantitative variables of the orofacial function. There were also no differences between the groups regarding the quantitative variables for orofacial function. 
Table 2. Result of Chi-square test for the comparison between quantitative variables of orofacial function and allocation into 3 groups

\begin{tabular}{|c|c|c|c|c|}
\hline \multirow{3}{*}{ Variable } & \multicolumn{3}{|c|}{ Groups } & \multirow{3}{*}{$\mathbf{P}$} \\
\hline & G1 & G2 & G3 & \\
\hline & $\mathrm{N}$ & $\mathbf{N}$ & $\mathrm{N}$ & \\
\hline \multicolumn{5}{|l|}{ Labial Posture } \\
\hline Closed & 46 & 36 & 31 & 0.461 \\
\hline Half-open or Open & 13 & 17 & 6 & \\
\hline \multicolumn{5}{|l|}{ Saliva } \\
\hline Controlled & 54 & 45 & 35 & 0.512 \\
\hline Excessive & 5 & 8 & 2 & \\
\hline \multicolumn{5}{|l|}{ Feeding difficulties } \\
\hline No & 44 & 34 & 29 & 0.194 \\
\hline Yes & 15 & 19 & 8 & \\
\hline \multicolumn{5}{|l|}{ Chewing } \\
\hline Normal & 45 & 39 & 29 & 0.905 \\
\hline Prolonged & 13 & 13 & 8 & \\
\hline \multicolumn{5}{|l|}{ GER } \\
\hline Yes & 51 & 40 & 28 & 0.180 \\
\hline No & 7 & 13 & 9 & \\
\hline \multicolumn{5}{|l|}{ Nasal reflux } \\
\hline Yes & 55 & 52 & 36 & 0.615 \\
\hline No & 3 & 1 & 1 & \\
\hline \multicolumn{5}{|c|}{ Coughing during feeding } \\
\hline Yes & 50 & 44 & 31 & 0.891 \\
\hline No & 8 & 9 & 6 & \\
\hline \multicolumn{5}{|c|}{ Non-nutritive Thumb sucking } \\
\hline Yes & 24 & 22 & 22 & 0.572 \\
\hline No & 6 & 9 & 5 & \\
\hline \multicolumn{5}{|l|}{ Breastfeeding } \\
\hline Yes & 6 & 7 & 6 & 0.437 \\
\hline No & 52 & 46 & 31 & \\
\hline \multicolumn{5}{|l|}{ Milk bottle } \\
\hline Yes & 16 & 13 & 11 & 0.851 \\
\hline No & 42 & 40 & 26 & \\
\hline \multicolumn{5}{|l|}{ Pacifier } \\
\hline Yes & 8 & 11 & 7 & 0.521 \\
\hline No & 21 & 19 & 30 & \\
\hline \multicolumn{5}{|l|}{ Speech delay } \\
\hline Yes & 54 & 50 & 32 & 0.369 \\
\hline No & 4 & 3 & 5 & \\
\hline \multicolumn{5}{|l|}{ Phonemic changes } \\
\hline Yes & 50 & 45 & 30 & 0.793 \\
\hline No & 8 & 8 & 7 & \\
\hline \multicolumn{5}{|l|}{ Choking } \\
\hline Yes & 49 & 47 & 49 & 0.540 \\
\hline No & 9 & 6 & 3 & \\
\hline
\end{tabular}

Note: GER: Gastroesophageal reflux N: Number of children; G1: Group 1; G2: Group 2; G3: Group 3; p: significance value for the chi-square test result, *: presence of statistical significance $(p<0.05)$. 
Table 3. Result of Kruskal-Wallis test for the comparison between quantitative variables of orofacial function and allocation into 3 groups

\begin{tabular}{ccccc}
\hline $\begin{array}{c}\text { Variable } \\
\text { (period in years) }\end{array}$ & $\begin{array}{c}\text { G1 } \\
\mathbf{M}( \pm \mathbf{S D})\end{array}$ & $\begin{array}{c}\text { G2 } \\
\mathbf{M}( \pm \mathbf{S D})\end{array}$ & $\begin{array}{c}\text { G3 } \\
\mathbf{M}( \pm \mathbf{S D})\end{array}$ & $\mathbf{P}$ \\
\hline Non-nutritive thumb sucking & $0.46( \pm 0.85)$ & $0.78( \pm 1.52)$ & $0.46( \pm 0.89)$ & 0.720 \\
Breast feeding & $1.46( \pm 1.34)$ & $1.58( \pm 0.98)$ & $1.10( \pm 1.18)$ & 0.646 \\
Bottle feeding & $2.25( \pm 1.95)$ & $3.06( \pm 2.07)$ & $2.86( \pm 2.03)$ & 0.865 \\
Use of pacifier & $2.96( \pm 1.54)$ & $2.76( \pm 1.83)$ & $2.95( \pm 1.35)$ & 0.967 \\
\hline
\end{tabular}

Note: G1: Group 1; G2: Group 2; G3: Group; M: Mean: SD: Standard Deviation; p: statistically significant for Kruskal-Wallis test.

\section{DISCUSSION}

This is the first study to observe whether the presence of $\mathrm{DOH}$ can directly interfere with the pulmonary function test. Notably, the maintenance of these habits can impair the structures and functions of the stomatognathic system, which are necessary for the performance of spirometry ${ }^{15}$. And although a direct relationship between the number of forced expiratory maneuvers and the history of $\mathrm{DOH}$ performed by the MBGR protocol has not been identified, this may have happened for some reasons that caused limitations in the study.

A first limitation of the study was the use of part of the MBGR protocol. Therefore, the entire protocol should be administered. A second limitation was the use of part of the protocol that consisted mostly of qualitatively evaluated items. However, in the literature, the effects of $\mathrm{DOH}$ have been evaluated mainly qualitatively with the MBGR protocol ${ }^{14,16}$.

Thus, specific SS assessment mechanisms are relevant, including the strength and tone of the facial musculature, as well as their respective functions. This analysis enables the understanding of the real contribution of each element of the SS in examinations involving correct placement and maintenance of a mouthpiece. In this context, electromyography verifies the activation of certain facial muscles that are used during the spirometry testings ${ }^{17}$ and could be included in further studies on the subject.

Some factors should be considered in order to achieve an acceptable spirometry maneuver: the examiner's adequate practical training, command voice, properly calibrated good quality equipment and control of the environment. In addition to these factors related to the technical aspects of the test, the understanding and cooperation of the patient to perform the maneuver adequately should also be considered, in order to avoid early glottis closure, early termination, glottal noise, buccal obstruction and airflow leak ${ }^{18}$.
Airflow leaks may be related to dentition. The correct positioning of the teeth in the mouthpiece ensures that leaks will not occur. But, especially in the age range in which the primary teeth will be replaced, the presence of spaces between the teeth can allow air to escape.

Among the various $\mathrm{DOH}$, oral breathing is a frequent habit among children, as reported in a study conducted by Melo and Pontes, showing occurrence of $48.60 \%$. They also suggest that the habit of breathing through the mouth can interfere with the growth of the facial skull, favoring some physical characteristics, namely, flaccidness face muscles, half-open lips, drooping cheeks, hypotonic tongue in the lower position or between teeth and dental malocclusion ${ }^{19}$.

Dental malocclusion, which may make the mouthpiece of the spirometer unstable, is often caused by $\mathrm{DOH}^{7}$. Its presence may be determinant for an oral respiratory pattern in some individuals, which may lead to alterations in the orofacial musculature, such as hypotonia and hypofunction of the mandibular elevator muscles $^{20}$.

Isometric contractions are mostly related to the alterations in the muscular strength (MS) and, therefore, to muscle fatigue ${ }^{21,22}$. They may interfere with the performance of a spirometry maneuver, since muscular action of the stomatognathic system is needed to seal the mouthpiece spirometer. A study conducted by Busanello-Stella et al. found a decrease in the muscular strength of the orbicularis oris muscles in children with nasal and oral breathing when sustaining isometric contraction against resistance. The group with mouth breathers reported earlier muscle fatigue ${ }^{21}$.

GER was one of the variables that, although not statistically significant, was very frequent among children who participated in this study. The literature shows that a GER's complication includes tooth erosion and it is associated with bruxism ${ }^{23,24}$. Thus, GER may alter SS functions and consequently interfere in the spirometry test. 
This study investigated the relationship between spirometry performance and $\mathrm{DOH}$ given the common difficulty faced by the pediatric age group to perform spirometry maneuvers adequately. This encouraged further researches that identified possible factors responsible for this event. In clinical practice, it is common for children to have spirometry curves with artifacts and parameters with values lower than those predicted for their age and gender. Coughing during the testing, mouthpiece obstruction, airflow leak, Valsalva maneuver and even unsatisfactory onset of forced spirometry maneuvers, with no evidence of effort and with inadequate termination, are some of the usual factors responsible for not meeting the criteria required for a valid the test. The relationship between these elements and $\mathrm{DOH}$, i.e., the hypothesis formulated in this study, has not been confirmed, and should be further investigated.

Comprehensive research should also be conducted on the relationship between history of $\mathrm{DOH}$ and specific evaluation of the orofacial musculature and the performance of spirometry maneuvers in children with respiratory impairment, such as asthma, allergic rhinitis and cystic fibrosis. In the study conducted by Carvalho-Oliveira et al., although on asthmatic adults, there was a high frequency of SS changes, which had an association with the severity of asthma observed by the forced expiratory volume in the first second $\left(\mathrm{FEV}_{1}\right)$ of spirometry test ${ }^{25}$. Campanha et al. performed speech therapy treatment and observed clinical and functional improvement in asthmatic children, evidenced by the increase in the percentage values of the spirometry parameters ${ }^{26}$.

\section{CONCLUSION}

There was no relationship between the presence of $\mathrm{DOH}$ and the number of forced spirometry maneuvers performed by healthy children in the study population. However, a comprehensive investigation of the SS is required. Furthermore, this study should be conducted in a pediatric population with respiratory impairment.

\section{REFERENCES}

1. Strini PJ, Barbosa TD, Gavião MB. Assessment of orofacial dysfunctions, salivary cortisol levels and oral health related quality of life (ORHQoL) in young adults. Arch Oral Biol. 2011;56(12):1521-7.

2. Bakke $M$, Bergendal $B$, McAllister A, Sjogreen $L$, Asten P. Development and evaluation of a comprehensive screening for orofacial dysfunction. Swed Dent J. 2007;31(2):75-84.

3. Cielo CA, Ribeiro VV, Christmann MK, Lima JPM, Pacheco-Rubim AB, Hoffmann $C F$ et al. Stomatognatic system changes in dysphonic individuals. Rev. CEFAC. 2016;18(3):613-25.

4. Garde JB, Suryavanshi RK, Jawale BA, Deshmukh V, Dadhe DP, Suryavanshi MK. An epidemiological study to know the prevalence of deleterious oral habits among 6 to 12 year old children. J Int Oral Health. 2014;6(1):39-43.

5. Suhani RD, Suhani MF, Muntean A, Mesaros M, Badea ME. Deleterious oral habits in children with hearing impairment. Clujul Medical. 2015;88(3):403.

6. Prado DGA, Sovinski SRP, Nary Filho H, Brasolotto $A G$, Berretin-Felix G. Oral motor control and orofacial functions in individuals with dentofacial deformity. Audiol Commun Res. 2015;20(1):76-83.

7. Sharma S, Bansal A, Asopa K. Prevalence of oral habits among eleven to thirteen years old children in Jaipur. Int J Clin Pediatr Dent. 2015;8(3):208.

8. Miller MR, Hankinson JA, Brusasco V, Burgos F, Casaburi $R$, Coates $A$ et al. Standardisation of spirometry. Eur Respir J. 2005;26(2):319-38.

9. Brazzale D, Hall G, Swanney MP. Reference values for spirometry and their use in test interpretation: A position statement from the Australian and New Zealand Society of Respiratory Science. Respirology. 2016;21(7):1201-9.

10. World Health Organization. The World Health Organization Quality of Life assessment (WHOQOL): position paper from the World Health Organization. Soc Sci Med. 1995;41:1403-9.

11. Solé D, Vanna AT, Yamada E, Rizzo MCV, Naspitz CK. International study of asthma and allergies in childhood (ISAAC) written questionnaire: validation of the asthma component among Brazilian children. Invest Allergol Clin Immunol. 1998;8(6):376-82.

12. Polgar G, Weng TR. The functional development of the respiratory system from the period of gestatin to adulthood. Am Rev Respir Dis. 1979;120(3):625-95.

13. American Thoracic Society. Standardization of spirometry - 2005. Eur Respir J. 2005;26:319-38.

14. Genaro KF, Berretin-Felix G, Rehder MIBC, Marchesan IQ. Avaliação Miofuncional Orofacial Protocolo MBGR. Rev. CEFAC. 2009;11(2):237-55.

15. Pereira TS, Oliveira F, Cardoso MCAF. Association between harmful oral habits and the structures and functions of the stomatognathic system: perception of parents/guardians. CoDAS [Internet]. 
2017 [cited 2017 Nov 05] ; 29(3):e20150301.

Available from: http://www.scielo.br/scielo. php? script $=$ sci_arttext\&pid=S2317 $17822017000300302 \&$ Ing=en. Epub May 15, 2017. http://dx.doi.org/10.1590/2317-1782/20172015301.

16. Johanns CM, Silvério K, Furkim AM, Marchesan I. Há relação de hábitos orais deletérios com a tipologia facial e a oclusão dentária? Rev. CEFAC. 2011;13(6):1095-102.

17. Prates LS, Gois M, Berwig LC, Blanco-Dutra AP, Busanello-Stella AR, Silva AMT. Clinical and electromyographic evaluation of mastication within different facial growth patterns. Rev. CEFAC. 2016;18(1):104-12.

18. Pereira CAC. Espirometria. J bras pneumol. 2002;28(3):1-82.

19. Melo PED, Pontes JRS. Deleterious oral habits in a group of children from a public school in Sao Paulo city. Rev. CEFAC. 2014;16(6):1945-52.

20. Emmerich A, Fonseca L, Elias AM, Medeiros UV. The relationship between oral habits, oronasopharyngeal alterations, and malocclusion in preschool children in Vitória, Espírito Santo, Brazil. Cad Saúde Pública. 2004;20(3):689-97.

21. Busanello-Stella AR, Blanco-Dutra AP, Corrêa ECR, Silva AMT. Electromyographic fatigue of orbicular oris muscles during exercises in mouth and nasal breathing children. Codas. 2015;27(1):80-8.

22. Buzinelli RV, Bérzin F. Electromyographic analysis of fatigue in temporalis and masseter muscles during continuous chewing. $J$ Oral Rehabil. 2001;28:1165-7.

23. Ranjitkar S, Smales RJ, Kaidonis JA. Oral manifestations of gastroesophageal reflux disease. J Gastroenterol Hepatol. 2012;27(1):21-7.

24. Mengatto CM, Dalberto Cda S, Scheeren B, Barros SG. Association between sleep bruxism and gastroesophageal reflux disease. J Prosthet Dent. 2013;110(5):349-55.

25. Carvalho-Oliveira M, Salles C, Terse R, D'Oliveira Júnior A. Association between severe asthma and changes in the stomatognathic system. J Bras Pneumol. 2016;42(6):423-8.

26. Campanha S, Fontes MJF, Camargos PAM, Freire LMS. The impact of speech therapy on asthma and allergic rhinitis control in mouth breathing children and adolescents. J pediat. 2010;86(3):202-8. 